\title{
HYPOPITUITARISM IN FIRST COUSINS
}

\author{
BY \\ DOUGLAS HUBBLE \\ From the Derbyshire Hospital for Sick Children and the Derbyshire Royal Infirmary
}

(Received for Publication January 8, 1951)

There is still much difficulty in elucidating the aetiological causes of dwarfism as it presents itself in children. After Erdheim in 1916 attributed dwarfism to a lack of pituitary secretion, it was for many years a common habit to implicate a pituitary deficiency in all dwarfs whatever their clinical features. A reaction to this unscientific approach was to be expected, and it has been so extreme in some quarters (Horstmann, 1950) that a pituitary origin for any type of dwarfism has been denied. Such a reaction is only possible if one contends that the enormous body of experimental work demonstrating the presence of an anterior pituitary growth hormone has no application to man, if one ignores the clinical pictures of dwarfism associated with destruction of the anterior pituitary, if one forgets the few reports of post-mortem examination in dwarfs which have demonstrated a deficiency of eosinophil cells in the anterior pituitary (Hewer, 1944), if one overlooks the clinical pictures of gigantism and acromegaly, and if one prefers not to remember that the absence of most hormones in the body is represented by a specific clinical syndrome. This aetiological nihilism, therefore, entails a denial of established fact which is too complete for most observers; but a moderate conservatism demands that some evidence of hypopituitarism should be forthcoming in any case of dwarfism before a diagnosis of pituitary dwarfism is made.

In the attempt to establish the aetiology of dwarfism in the individual child, all the systemic causes of infantilism and all the bone diseases which can produce short stature must be excluded. There are then two questions which urgently require an answer, Is this child suffering from hypothyroidism ? Is there organic disease in the region of the pituitary? The clinical picture of cretinism is well recognized, but continued observation may be necessary to establish the diagnosis of juvenile myxoedema. If an arrest of growth can be shown not to be due to disease or malnutrition, then hypothyroidism should be suspected, and the signs of diminished vitality, cold extremities, slow pulse, electrocardiographic abnormalities, raised blood cholesterol level, and low basal metabolic rate must be looked for. Gross destruction of the pituitary by tumour, by xanthomatosis or by inflammation is rare in children, and a craniopharyngioma as a cause of dwarfism is very uncommon. When these infrequent causes are excluded, there remains a large group of children in whom a cause for their dwarfism has still to be sought, and among them will be examples of pituitary dwarfism.

The separation of the cases of pituitary dwarfism due to the absence of the growth hormone would be simple if it were possible to assay the level of growth hormone in the blood or to determine the quantity of its metabolized products in the urine. However, no such investigations are as yet possible, and to determine whether dwarfism is related to a deficiency of the growth hormone, it is necessary to depend on a clinical picture of pituitary dwarfism, and to look for evidence of the absence of the other pituitary hormones. The gonadotropic hormones are excreted in small quantities before puberty. Until the age of puberty arrives, and the average time of onset may extend from 10 to 15 years of age in girls and 11 to 16 years in boys, it is not possible to support a diagnosis of pituitary dwarfism by the absence of gonadotropic hormones whether directly or indirectly.

The remaining two pituitary hormones are the thyrotropic and the adrenocorticotropic hormones. It is rarely possible to demonstrate any evidence of thyroid underaction in the absence of frank cretinism or myxoedema. Evidence of defective adrenocortical function should be sought in disturbances of carbohydrate metabolism, as in hypoglycaemia which may be due to a deficiency of the gluconeogenetic hormones of the adrenal cortex; in a disturbance of serum electrolytes together with 
a positive Robinson-Power-Kepler test; and, as the age of puberty approaches, in the absence of sexual hair in both sexes and by a low output of 17-ketosteroids in males. It should be remembered, however, that such disturbance of carbohydrate metabolism may be associated with the absence of the growth hormone which inhibits the secretion of insulin in rats (Anderson and Long, 1947), thereby causing hyperinsulinism; that a disturbance of electrolytes can seldom be demonstrated in pituitary dwarfism; and that the signs of androgen deficiency are not apparent till puberty approaches. A deficiency of the anterior pituitary or the adrenal cortex may be demonstrated by Thorn's adrenalineeosinophil test, while an adrenocortical defect may be detected by the absence of both the normal fall in the eosinophil count, and the normal rise in the urinary uric acid/creatinine ratio after $25 \mathrm{mg}$. of ACTH.

It is probable, however, that deficiency of the growth hormone may occur alone and in the absence of coincident deficiency of the other anterior pituitary hormones. This observation is supported by some clinical evidence, but also by an occasional pathological report. Hewer (1944) recorded an extensive necropsy examination in a woman aged 76 , whose height was $3 \mathrm{ft} .11 \mathrm{in}$. and who had borne one child, and in the anterior pituitary of this dwarf he demonstrated an almost complete absence of eosinophil cells. In a child, therefore, in whom dwarfism exists before the age at which the sex hormones can be expected to be active, or in whom dwarfism continues after puberty, there usually remains a considerable diagnostic problem as to the aetiology of the dwarfism. Other causes of dwarfism, both constitutional and genetic, must then be considered before a firm diagnosis of pituitary dwarfism can be made. In dwarfs in whom epiphyseal development occurs at the average age it appears improbable that an absence of the growth hormone can be implicated. Where an absence of the growth hormone can be assumed, epiphyseal development will show moderate retardation. The group with normal epiphyseal development will include the so-called ' primordial ' dwarfs who occur sporadically in families whose members are of normal height; it will also include children of inherited short stature.

It is useful to recall Bauer's (1943) diagram which illustrates the aetiology and pathogenesis of developmental disorders. Disturbances of growth may be caused in A (' a mess in the chromosomes'), in C (pituitary, thyroid, adrenal, or testis), in B (skeletal disorders or diminished sensitivity of end organ), and in $\mathrm{D}$ (hypothalamus) while they may be mediated through paths $1,2,3,4$, and 5 .

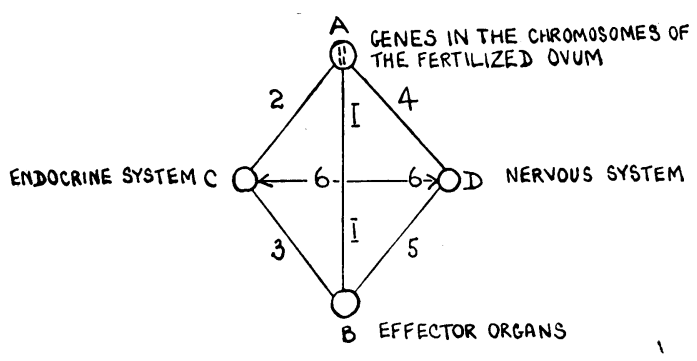

It must be assumed that the 'primordial ' dwarfs grow to a height genetically determined; whether or not these primordial dwarfs are an example of a diminished sensitivity of the end organ, in this case all the somatic tissues, in the presence of normal or increased amounts of the specific hormone, cannot be decided in the absence of assays of the growth hormone. The most commonly occurring example of the primordial dwarf is the so-called Lorain dwarf. Here the height age is less than in hypopituitary dwarfism, but the bone age is within average limits. In other respects, except in a failure to grow, development is perfect and there is no persistence of the infantile facial appearance. The group of genetic dwarfs also includes those girls and women who suffer from ovarian agenesis in association with other congenital defects. In the group of dwarfs showing delay of epiphyseal development and fusion (hypothyroidism already excluded) are the pituitary dwarfs and also children whom Wilkins (1950) described as showing delayed adolescence. This last group show a consistent delay of two to four years in their epiphyseal development, and in their early 'teens fail to show the normal spurt in growth and sexual development. The spurt comes at a late age, and they may never attain average height. It appears probable that this is a heterogeneous group, in some of whom the peculiar pattern is genetically determined, while in others the delayed growth is the result of environmental factors. In either case, the delayed epiphyseal development may indicate relative deficiency of the growth hormone or diminished sensitivity of the skeleton to its stimulus. The genetic cases may be associated with a disturbance of the normal growth hormone: androgen ratio.

Talbot (1945), and Talbot, Sobel, Burke, Lindemann, and Kaufman (1947) have suggested that malnutrition, sometimes resulting from emotional anorexia, may be an environmental factor in the defective formation of the growth hormone.

In our present state of diagnostic inadequacy, any evidence which supports a diagnosis of pituitary dwarfism is therefore valuable. The description follows of two boys who were first cousins, born of 




FIG. 2.

FIG. 2.-T.B. Note immature facies.

FIG. 1.-T.B., aged $7 \frac{1}{2}$ years, height $37 \frac{1}{2}$ in., photographed with boy of the same age.

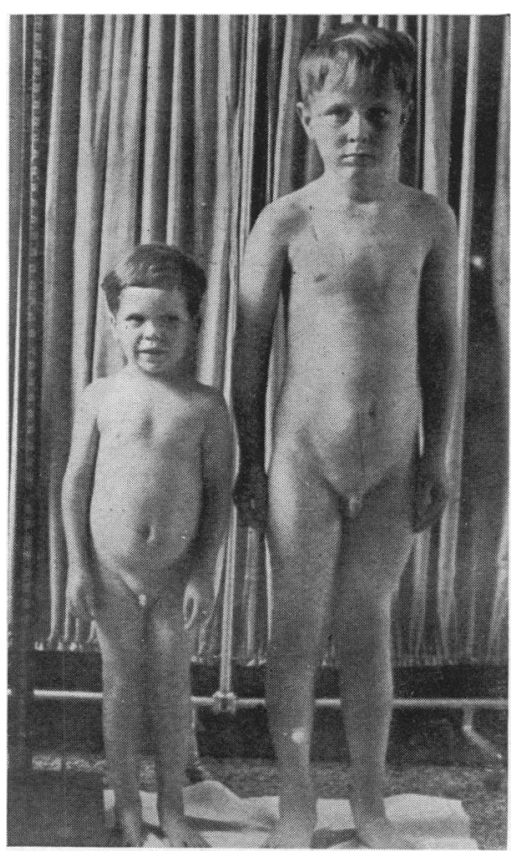

FIG. 1,

two sisters, whose husbands were unrelated to each other or to their wives. The boy, aged $7 \frac{1}{2}$ years when first seen, showed the clinical picture of pituitary dwarfism, and his male cousin, aged 20 years, showed hypogonadism with a eunuchoid habitus, delayed epiphyseal fusion, osteoporosis, and deficiency excretion of urinary gonadotropins.

\section{Case Reports}

Case 1. Terence B., aged $7 \frac{1}{2}$ years (Figs. 1 and 2), weighed $7 \mathrm{lb}$. at birth. He developed normally during the first two years of his life, reaching his milestones at an average age. His mother did not notice that he was not growing naturally until he was 2 years old. He was then treated by thyroid tablets for two years, but no improvement in growth occurred. He was taken to another city where he was given other treatments without success.

His mental age was within average limits and he was doing reasonably well at school.

He had had pertussis and measles but no other infectious diseases.

All the members of the family are of normal growth and sexual development, except Terence's first cousin D.T. described below.

On examination Terence B. proved to be an alert, happy child, with normal functions and with a good appetite. The facies shows an infantile naso-orbital configuration. The skin was not pale, and the extremities not cold. His height was $36 \frac{1}{2}$ in. (normal approximately $47 \frac{1}{2}$ in.). The span was 35 in. The height age was less than 3 years. His weight was $30 \mathrm{lb}$. (normal approximately $50 \mathrm{lb}$.). The upper segment was $19 \mathrm{in}$., the lower segment $17 \frac{1}{2}$ in., and the ratio of the segments $1 \cdot 1: 1$ (normal 1.1:1). The head circumference was $19 \frac{1}{2}$ in., the chest circumference $19 \frac{1}{2}$ in.

A radiograph of the bones showed that the pituitary fossa was normal. There was no epiphyseal dysgenesis. All bones of the carpus (except the pisiform), and the head of the radius and patella were present. The distal epiphyses of the ulna (Fig. 3) and internal epicondyle of the humerus were absent. Bone age was therefore between 5 and 6 years.

The insulin sensitivity test (Fig. 4) showed a normal sensitivity with some lack of hypoglycaemic response:

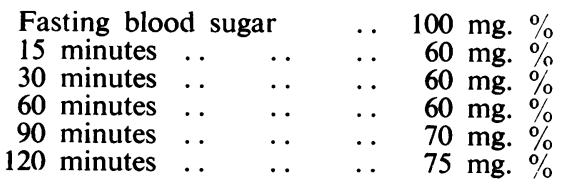

The blood count was normal. W.B.C.s were 11,600 per c.mm. (eosinophils $3 \%$ ). The response of eosinophils to $0.3 \mathrm{mg}$. adrenaline was not abnormal.

The blood cholesterol level was $281 \mathrm{mg}$. \%. This was repeated one month later (not after thyroid therapy) and was then $241 \mathrm{mg}$. \%.

The electrocardiogram was normal.

The Wassermann and Meinicke reactions were negative.

The Mantoux test ( 1 in 1,000 O.T.) was negative.

The urinary hormonal excretion (Dr. A. M. Hain) showed urinary 17-ketosteroids $3.9 \mathrm{mg}$. in 24 hours.

All other investigations, both radiological and biochemical, gave normal results.

In view of Terence B.'s high blood cholesterol level and despite the absence of any other signs of hypothyroidism, he was treated for three months by thyroid therapy. There was no acceleration of growth 
FIG. 3.-Radiograph of carpus of T.B.; note absence of ulnar epiphysis which normally appears at the age of 6 years.
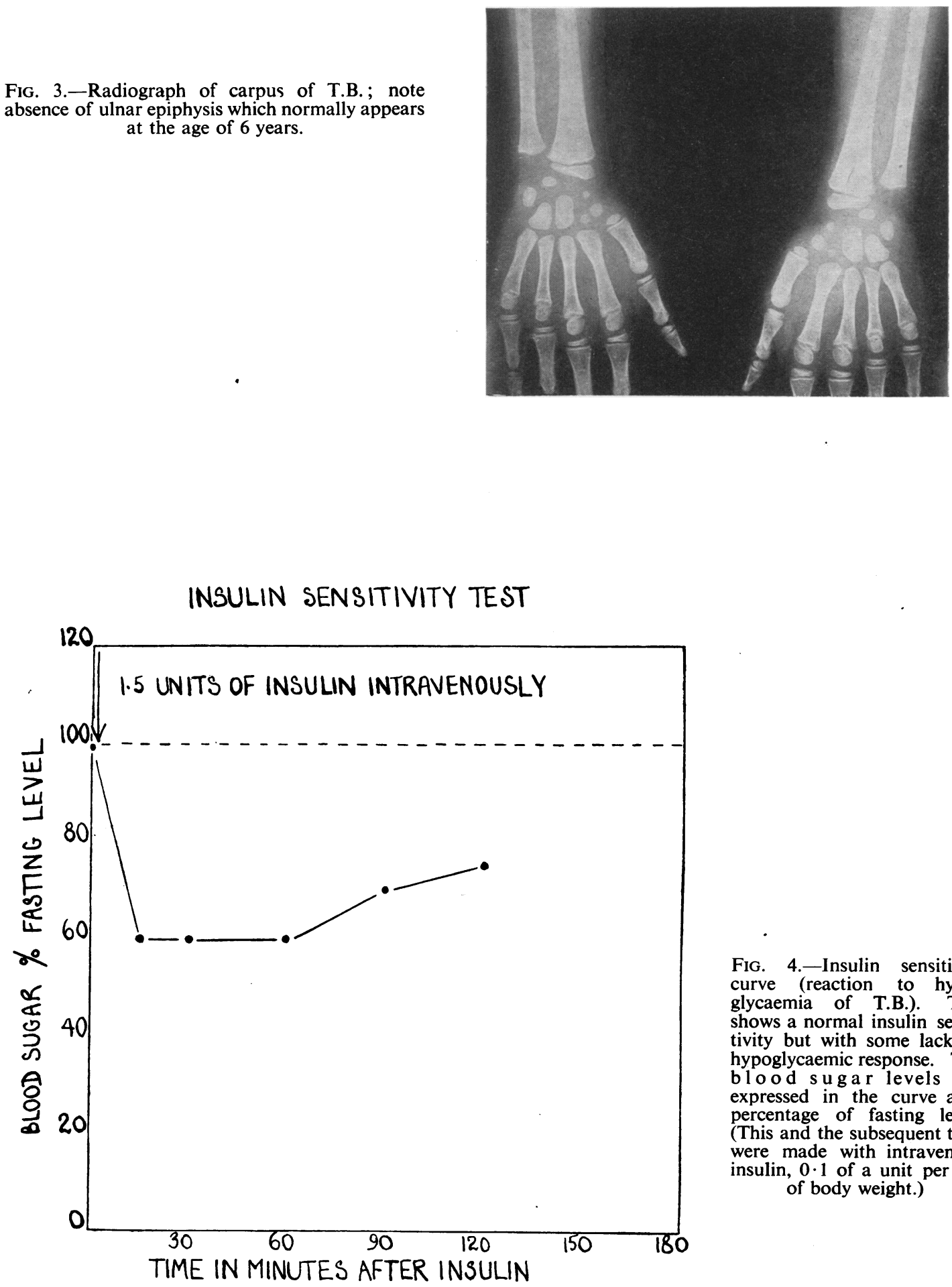

FIG. 4.-Insulin sensitivity curve (reaction to hypoglycaemia of T.B.). This shows a normal insulin sensitivity but with some lack of hypoglycaemic response. The blood sugar levels are expressed in the curve as a percentage of fasting level. (This and the subsequent tests were made with intravenous insulin, $0 \cdot 1$ of a unit per $\mathrm{kg}$. of body weight.) 
and no advancement of bone age. He was then treated for three months by growth hormone, ' antuitrinG' (Parke Davis), with no improvement. For a further three monthis he was given 'amdinon' (Organon), a preparation of thyrotropic hormone with growth hormone; this also produced no appreciable growth. During the next three months he was given methyltestosterone therapy and grew $1 \frac{1}{2}$ in. in this period.

Case 2. D.T., first cousin to T.B., aged 20, has never shaved, and his voice is said to have become no deeper since his childhood. He had had no penile erection and no seminal emission before admission to hospital. He was referred to the hospital for treatment of varicose veins.

The family history shows that there were no siblings in the family. Both father and mother are of normal growth and sexual development.

On examination the patient's weight was 9 st. $2 \mathrm{lb}$.; his height $5 \mathrm{ft} .7 \mathrm{in}$. The span was $5 \mathrm{ft}$. 11 in.; the upper segment was 31 in., the lower segment 36 in., and their ratio $0 \cdot 86: 1$. The body was of feminine type with wide hips (Figs. 5 and 6). There was sparse axillary and pubic hair, and no hair on the face. Both testes and penis were much smaller than the average for an adult male. He had genu valgum with pes planus, and there were varicose veins in both legs.

The dermatological report (Dr. P. D. C. Kinmont) showed mild xeroderma of the legs and face with a probable allergic eczema on cheeks, forehead, arms, and ankles. He had very lax joints, particularly in the feet, with curious circular scars on the legs and feet. The appearance was suggestive of the scars of epidermolysis bullosa. There was no cutis laxa hair. He had a tendency to bruising, and there was capillary dilatation on the legs. No other abnormal sign was discovered on clinical examination in any other system.

Investigation gave the following results: serum calcium level 10:4 mg. \% (after calcium and testosterone

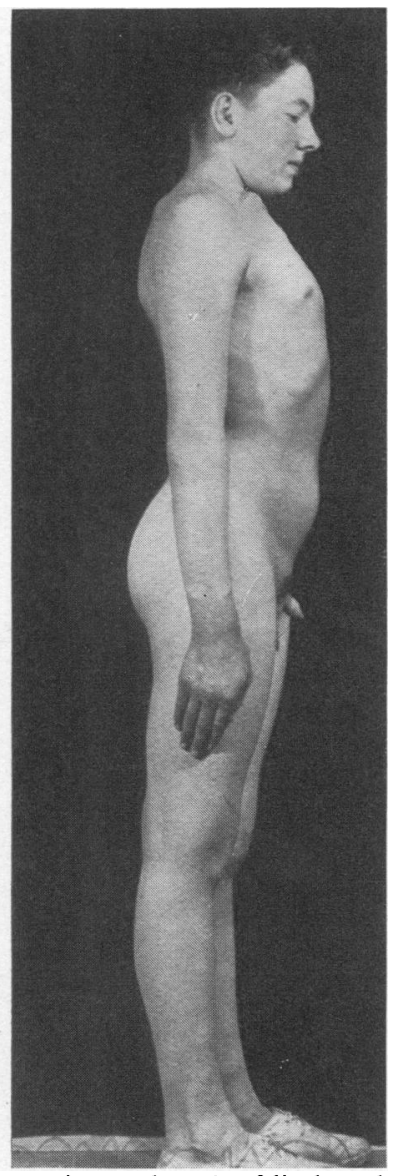

therapy $11.8 \mathrm{mg} . \%$ ); serum phosphorus level $5.0 \mathrm{mg}$. \%; alkaline phosphatase level $5 \cdot 2$ units; serum proteins 7.6 g. $\%$ (serum albumin $4.4 \mathrm{~g}$. $\%$, and serum globulin $3 \cdot 2$ g. \%).

The insulin sensitivity test (Fig. 7) gave the following results:

\section{Fasting}

blood sugar $100 \mathrm{mg} . \%$ 30 minutes $60 \mathrm{mg} . \%$ 60 minutes $80 \mathrm{mg} . \%$ 90 minutes $80 \mathrm{mg} \%$ 120 minutes $90 \mathrm{mg} . \%$

No excess of fat was found in the chemical examination of a three-day specimen of faeces.

Analysis of urinary 17-ketosteroids (Dr. A. M. Hain) gave: on June 16, 1950, 10.2 mg. in 24 hours; on June 26, 1950, 9.1 mg. in 24 hours. After two months' methyltestosterone therapy, on August 27, 1950, excretion was raised to $14.0 \mathrm{mg}$. in 24 hours. The urinary gonadotropins on May 16, 1950, were less than 6 M.U. in 24 vide hips. 
INSULIN SENSITIVITY TEST

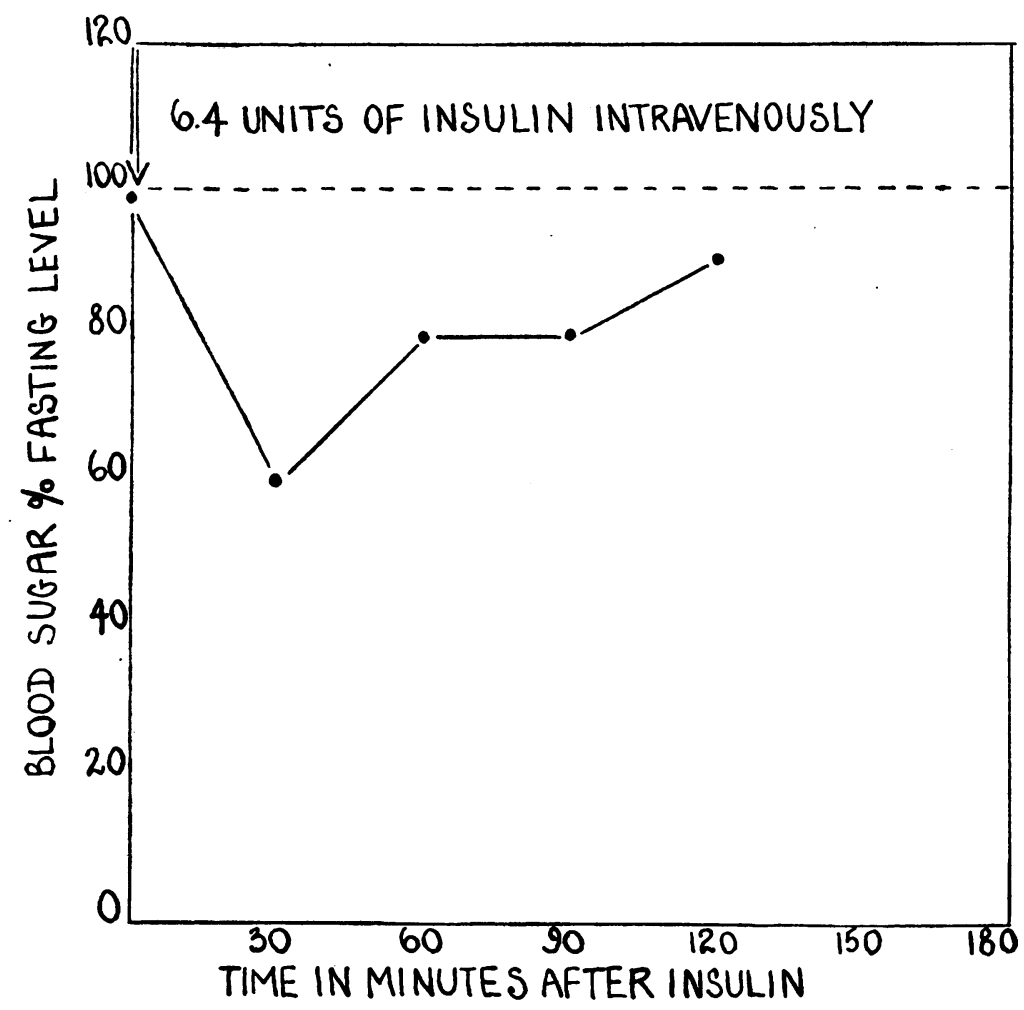

FIG. 7.-Insulin sensitivity curve (reaction to hypoglycaemia of D.T.).

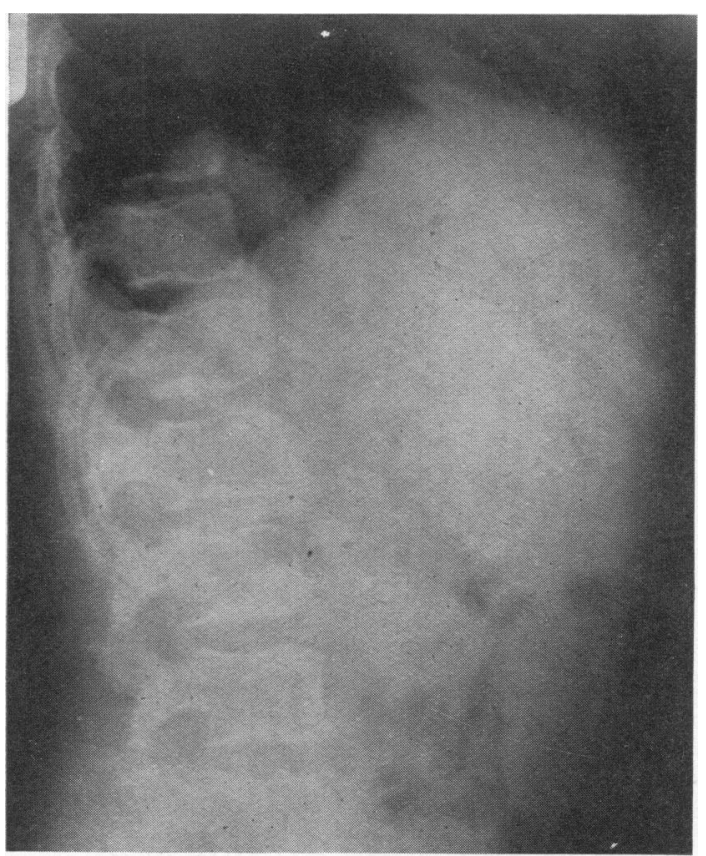

FIG. 8.-Radiograph of lumbar spine of D.T. showing osteoporosis. 


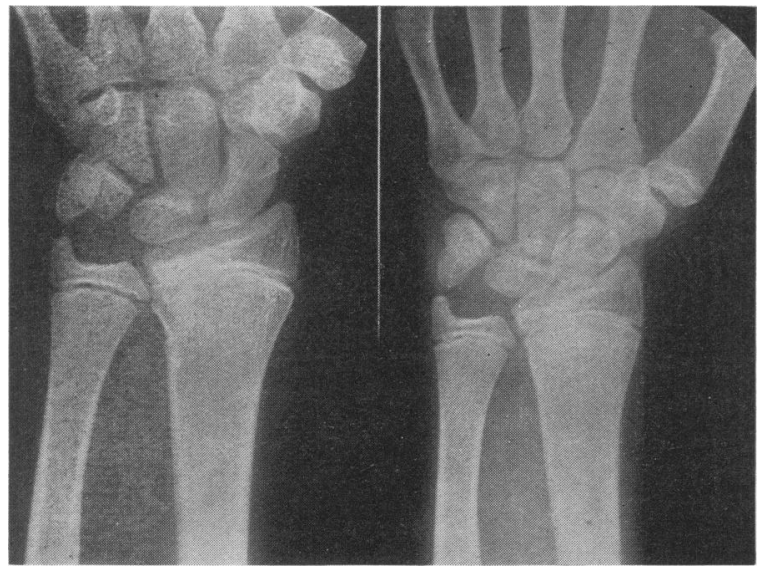

Fig. 9.-Ulnar epiphysis of D.T. not yet united.

FIG. 10.-Photomicrograph of biopsy of testis of D.T. $\times 900$.
Treatment by methyl-testosterone $(20 \mathrm{mg}$. b.d.) caused increased growth of axillary and pubic hair. The facial hair grew, and it became necessary for the patient to shave once a week. The penis increased in size, but the testicles showed no alteration in size. He had occasional erections, but no seminal emission as a result of treatment. His voice deepened and both his habitus and his outlook became more masculine.

\section{Discussion}

The boy T.B., aged $7 \frac{1}{2}$ years, showed the clinical appearance of a pituitary dwarf, but the diagnosis of pituitary dwarfism should not be considered probable unless (1) cretinism or myxoedema is excluded, (2) the height is at least $20 \%$ below the average for coevals, (3) the bone age, after the age of 5 years, is at least two years behind coevals, (4) the ratio of upper to lower segment corresponds approximately to the 'chronological' age (proportionate dwarf), and (5) the features are immature. If these criteria are present, then it is justifiable to assume that the dwarfism is hypopituitary in origin whether the defect is congenital or conditioned. It may well be that growth retardation of lesser

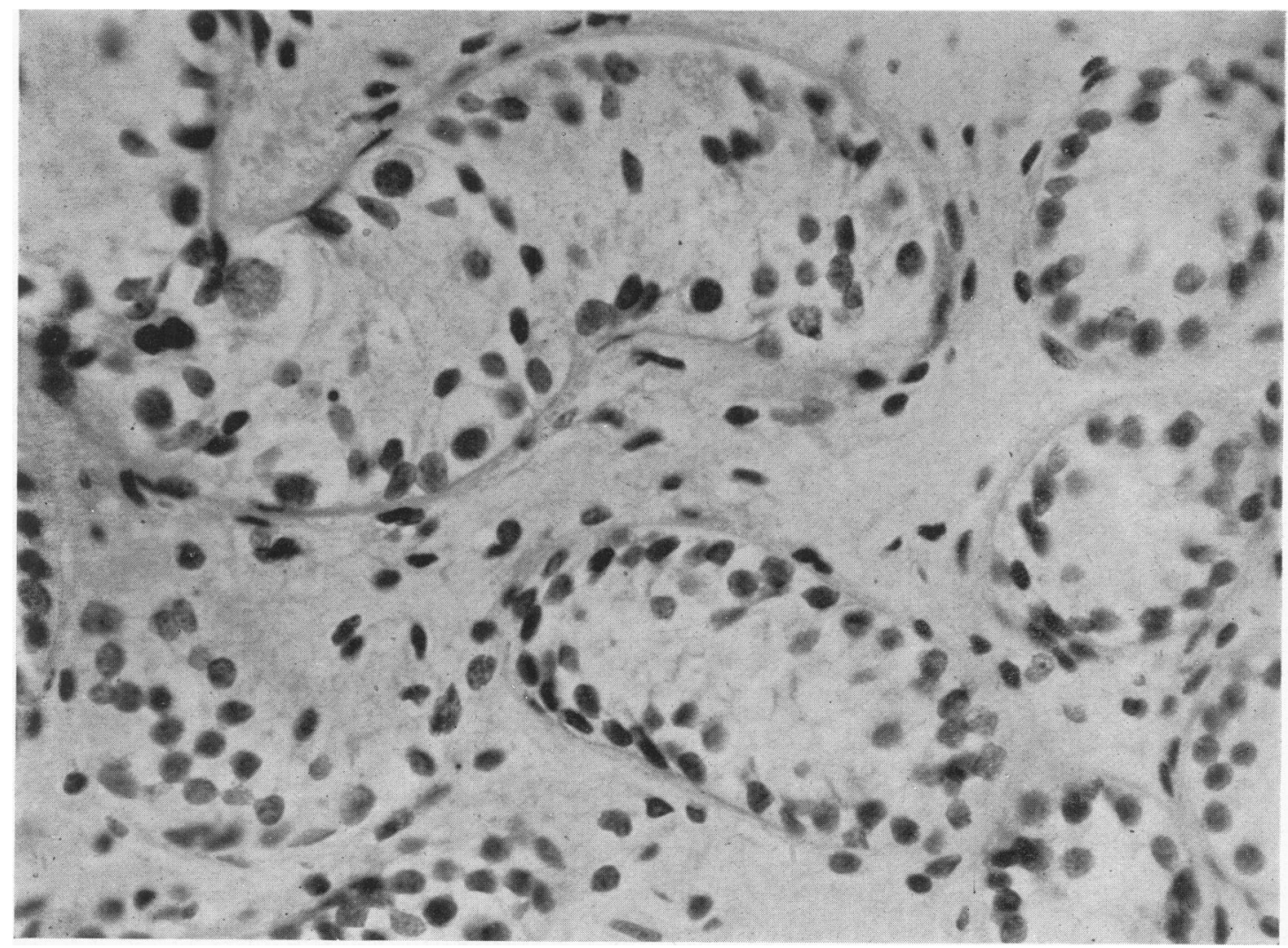


degrees may occur with partial deficiency of the growth hormone, but this cannot be established in the absence of evidence of deficient production of anterior pituitary hormones whether before the age of puberty or after. It has been postulated by some observers that the absence of growth hormone produces defective bone growth by conditioning an absence of thyrotropic hormone, while others have suggested that there are reasons for thinking that the dwarfism of hypothyroidism may be due to a conditioned deficiency of growth hormone. These theories are speculative, and as a rule in pituitary dwarfs there is no evidence of hypothyroidism, a diagnosis which in children can be made with considerable exactitude. In T.B. there was no evidence of hypothyroidism except for a raised serum cholesterol level, and growth was not accelerated by thyroid therapy. A clinical diagnosis of pituitary dwarfism in T.B. is supported by finding hypopituitarism in his first cousin, D.T., with evidence of hypogonadism (testicular hypoplasia), eunuchoid habitus, and osteoporosis with delayed epiphyseal fusion.

The diagnosis of hypopituitarism in D.T. is confirmed by the low gonadotropin urinary excretion (less than 6 M.U. while the normal is 6-50 M.U. in 24 hours). The excretion of 17-ketosteroids (9-10 mg. in 24 hours before testosterone therapy) represents the normal product of adrenal androgens. The scarcity of Leydig cells in the testis gave histological support to the clinical diagnosis of absent testicular androgens (representing one-third of the 17-ketosteroid excretion) as evidenced by the eunuchoid habitus (low upper to lower segment ratio and span greater than height), the sparseness and absence of sexual hair, the delayed epiphyseal fusion and the generalized osteoporosis. Osteoporosis of the degree seen in D.T.'s skeleton is rare in hypopituitarism, although it is well explained by the absence of testicular androgens (Albright and Reifenstein, 1948). In their absence there is a defect in the deposition of osteoid tissue while resorption of bone proceeds normally.

The insulin sensitivity test was normal in both subjects, except that in T.B. there was some diminished response to hypoglycaemia. Although this was shown to be present in subsequent tests, it is doubtful if it can be interpreted as evidence for the absence of an anti-insulin factor (? growth hormone).

The purified growth hormone causes neither nitrogen retention in normal individuals nor does it enhance growth in pituitary dwarfs (Bennett, Weinberger, Escamilla, Margen, Li, and Evans, 1950) and yet no inhibitors to its action in the blood were demonstrated by these observers. The most probable explanation is that in man it requires some catalyst as yet undetermined for its effective action. It would be premature to use the failure of the growth hormone in therapy as an additional argument against a hypopituitary aetiology for dwarfism in man.

Treatment by thyroid therapy, by thyrotropic hormone and by growth hormone (" antuitrin-G' Parke Davis) in T.B. produced no result; treatment by chorionic gonadotropins in D.T. produced no enlargement of the testes. Treatment by methyltestosterone in T.B. caused acceleration of growth, while in D.T. increased density of bone and growth of sexual hair could be demonstrated after methyltestosterone therapy. In D.T., since chorionic gonadotropins failed, treatment by methyl-testosterone was indicated. It will develop the secondary sexual characters of the male, and it will retain nitrogen and calcium and increase the density of bone. It has been suggested by Talbot et al: (1947) that methyl-testosterone will stimulate the anterior pituitary, and there is evidence that this effect was achieved in D.T., for after two months' therapy with methyl-testosterone the excretion of urinary 17 -ketosteroids increased from 10 to $14 \mathrm{mg}$. in 24 hours. Testosterone in the experimental animal inhibits both the gonadotropic hormones and ACTH without affecting the thyrotropic hormone, but these effects may be altered by a change of dosage. Since there is no therapeutic preparation of the follicle stimulating hormone and lateral hormone, there is no possibility of stimulating the testis by any other means.

The advisability of the treatment of pituitary dwarfism in children before puberty by testosterone is debatable. The arguments against it are that the testes may thereby be damaged, and that the ultimate height of the child will not ke increased by such therapy. It is possible to damage the testis after puberty by testosterone therapy, but there is no evidence that this occurs before puberty. In men after daily doses of $25 \mathrm{mg}$. of testosterone propionate over long periods (24-91 days) it has been shown that the testicular changes are completely reversible (Heller, Nelson, Hill, Henderson, Maddock, and Jungck, 1950). There is one biopsy report in the literature (Talbot, 1945) in which testosterone was administered to a boy in interrupted courses between 13 and 16 years of age, and testicular biopsy at 16 years showed no evidence of damage. There can be no proof that testosterone will not increase the ultimate height of a pituitary dwarf. Providing it is not used in children whose bone age is normal (e.g. non-pituitary dwarfs), and providing the child's bone age is not accelerated beyond the normal for its age, it seems reasonable to employ 
testosterone in therapy, using it in interrupted courses, and with adequate radiographical control.

\section{Summary and Conclusions}

The difficulties of distinguishing pituitary dwarfs before the age of puberty from the other types of dwarfism - whether ' primordial,' genetic or acquired -are considered.

Criteria are suggested for the clinical diagnosis of pituitary dwarfism of which the most important are the persistence of an immature facies, the retardation of bone age by at least two years, and a height which is at least $20 \%$ below the average for coevals in the proven absence of hypothyroidism.

Two male first cousins are described, in one of whom, aged $7 \frac{1}{2}$ years, pituitary dwarfism was present and in the other, aged 20 years, pituitary hypogonadism, with deficient urinary excretion of gonadotropins, testicular hypoplasia, eunuchoid habitus, defective secondary male sexual characters, osteoporosis with retarded union of epiphyses, could be demonstrated.

It is suggested that when other treatments have failed it is justifiable to use methyl-testosterone therapy after the age of puberty in pituitary hypogonadism, and before the age of puberty in pituitary dwarfism. In the latter case care must be exercised not to advance the child's bone age beyond the chronological age so that early epiphyseal closure is avoided.

\section{REFERENCES}

Anderson, E., and Long, J. A. (1947). Endocrinology, 40, 98 .

Albright, F., and Reifenstein, E. C. (1948). "The Parathyroid Glands and Metabolic Bone Disease.' Baltimore.

Bauer, J. (1943). 'Constitution and Disease,' p. 52. London.

Bennett, L. L., Weinberger, H., Escamilla, R., Margen, S., Li, C. H., and Evans, H. M. (1950). J. clin. Endocrinol., 10, 492.

Erdheim, J. (1916). Beitr. path. Anat., 62, 302.

Heller, C. G., Nelson, W. O., Hill, I. C., Henderson, E., Maddock, W. O., and Jungck, E. C. (1950). J. clin. Endocrinol., 10, 816.

Hewer, T. F. (1944). J. Endocrinol., 3, 397.

Horstmann, P. (1950). Acta. med. scand. Suppl., 239, p. 383.

Talbot, N. B. (1945). Bull. New Engl. med. Center, 7, 117.

- and Sobel, E. H. (1947). Advanc. Pediat., 2, 238. , Burke, B. S., Lindemann, E., and Kaufman, S. B. (1947). New Engl. J. Med., 236, 783.

Wilkins, L. (1950). 'The Diagnosis and Treatment of Endocrine Disorders in Childhood and Adolescence.' Springfield, Illinois. 